\title{
Nanotechnology and textiles: A perspective on defense applications
}

\author{
Mangala Joshi \\ Department of Textile Technology, Indian Institute of Technology Delhi \\ Email:mangala@textile.iitd.ac.in,mangalajoshi9@gmail.com
}

The use of nanomaterials is growing at a tremendous rate to enhance the performance properties of polymers used in various fields including textiles. The defense application is one such area where the potential of nanotechnology is being utilized through constant research and development.

Polymer nanocomposites are a unique new class of materials with an ultrafine dispersion of nanomaterials in a polymeric matrix. They exhibit much superior properties in terms of increased strength and modulus, improved heat resistance, decreased gas permeability and flame retardance at very low loadings of $<5$ wt $\%$ of nanofillers, as compared to neat polymers or conventional composites. Thus they show promising applications in developing advanced textile materials such asNanocomposite fibers, nanofibers and other nanomaterial incorporated fibers and coated textiles for applications in medical, defense, aerospace and other technical textile applications such as filtration, protective clothing besides a range of smart and intelligent textiles.

Our research group at Textile Department, IIT Delhi, has undertaken a lead in this area and several R\&D projects sponsored by MHRD, DST (Nano-Mission), DRDO and Industry have been taken up in last 8-9 years. This paper discusses highlights of our research efforts being done in the area of "Polymer Nanocomposites" as an advanced material in developing high performance textiles having potential applications in Defense Sector.

Polymers nanocomposites offer tremendous potential when produced in fiber form and offer properties that leapfrog those of currently known commodity synthetic fibres. We have investigated nanocomposite fibers based on all the three major types of nanofillers viz layered silicate nanoclays (MMT), carbon nanotubes (CNT) and nanofibers, nanographite / graphene and hybrid nanostructured materials such as POSS. These nanocomposite fibers show superior mechanical, thermo - mechanical, UV
\& creep resistance and improved dyeability and very low loadings of nanomaterials.

Another project of importance is 'Polymer Nanocomposite Coated Textiles for Inflatables' sponsored by ADRDE, DRDO, Agra. The use of nanoclay is known to significantly reduce the gas barrier property of nanocomposites. This property has been used to develop nanocomposite coatings for inflatables and balloons used for surveillance purposes. These nanocomposite coatings reduce the tear weight of the balloon and as the gas permeability is reduced the float time is likely to increase.

The hybrid nanoparticle based polymer coatings can increase the shielding effectiveness against radar for camouflage fabrics and nets. The flat attenuated response over wide radar frequency range achieved by these kinds of microwave absorbent nanocomposite has the potential for application in camouflaging various defense establishments as demonstrated by the work being done by our research group [1].

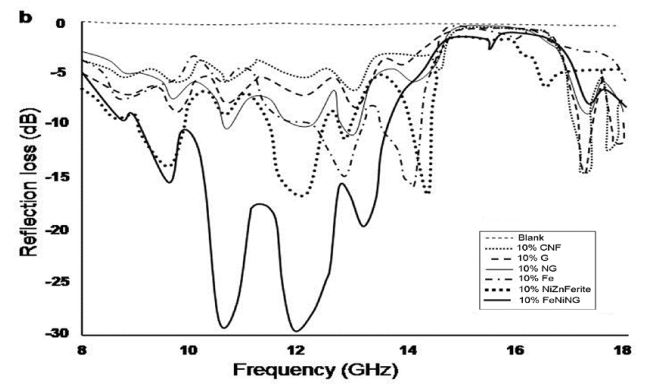

Figure 1: Microwave refection loss of different coated samples at high frequency range $(8-18 \mathrm{GHz})$. FeNG, iron-coated nanographite; FeNiNG, ironnickel nanographite; NG, nanographite; G, graphite; NiZnFerrite, nickel-zinc ferrite [1]

\section{Reference}

1. A. Bhattacharyya \& M. Joshi, Functional Properties of Microwave Absorbent Nanocomposite Coatings based on Thermoplastic Polyurethanebased and Hybrid Carbon-based Nanofillers, Polymers for Advanced Technologies, 23 (2012), 975-983. 